has been the last to close, or in other words, that side on which the testicle was the last to descend, is the side on which we usually have the rupture occurring; and knowing that the right testicle is generally the last to descend, we naturally find that hernia in infants is also most frequently observed on this side. That the rupture occurs on the side on which the testicle was the last to descend, is only what one would suppose, for the uniting medium, which is effecting a closure of the canal on this side, is not in so advanced a condition of organisation as on the other side, where the testicle has taken its place prior to the other.

It is thus easily seen how a single truss frequently produces a double rupture. The cause of the obstruction to the outflow of urine is still present in the phimosis; and, one inguinal canal being guarded by the single truss, the abdomen gives way at its next weakest point, viz., the other inguinal canal, and a double rupture is the consequence. Such a result might have been prevented by early circumcision. The hernia in these cases is generally scrotal, or, if not, it soon becomes so by the wedge-like projection of the intestines; and whether it be congenital or infantile in variety, depends upon the amount of the funicular process of peritoneum which has become converted into fibro-cellular tissue, or which has been broken down by the aforesaid propulsion of intestine. The operation of circumcision as performed upon young children, and which was done in all of the cases of which I previously spoke, is both easy of performance and effective in its results ; easy of performance, because no sutures are ever required, children bear pain well, and the parts are usially well in a week or ten days; it is effective in its results, because the hernia then stands every chance of being effectually cured by the application of a truss, the exciting cause having been removed; and at all events a double rupture is prevented by its early adoption. Occasional difficulty arises in the operation when the rupture is very large, for the penis, buried by the projection of the rupture, is represented in such cases by a button-hole aperture, and the operation is then more easily effected if the rupture be commanded by a double inguinal truss during its performance. In conclusion, I would suggest that, whenever an elongated or contracted prepuce is present in infants, the sooner circumcision is performed the better; thereby the more serious complaint of rupture would be prevented.

\section{THE SALICYLATES.}

BI PROSSER JAMES, M.D.,

Lecturer on Materia Medica in the London Hospital Medical School; Physician to the Hospital for Diseases of the Throat and Chest, etc.

Professor Charteris seems (JoUrnal, February i2th) to have abandoned salicylate of soda after a single trial; but the cases reported in the JOURNAL of January 29th show that it is being freely used in hospital practice. Perhaps the early introduction of this salt, and its ready solubility, as well as its sweetish taste, have combined to make it the favourite. I should, however, be glad to introduce to the profession some others which are deserving of their attention. In acute rheumatism, salicin seems likely to maintain its pre-eminence, because in this disease it is necessary to bring the patient rapidly under the influence of the remedy. This can only be accomplished by full doses at frequent intervals; and salicin seems to be more readily tolerated than salicylic acid or the salicylates; indeed, very disagreeable, not to say dangerous, symptoms arise with these salts, as testified by Dr. Charteris and many others. Where, however, it is not necessary to saturate the system quickly with the drug, no such disagreeable effects will occur, and the salicylates of soda and other bases deserve a more extensive trial. All produce similar effects, so far as the acid is concerned, each giving rise to variations due to its respective base.

Salicylate of Ammonia.-When it is merely desired to obtain a freely soluble and diffusible combination of salicylic acid ammonia may be substituted for soda, and the salt thus obtained administered in corresponding quantites; the only differences in the effects being due to the change of base.

Salicylate of Potash may be first named as a substitute for the soda salt; it is similar in taste, very soluble, and may be administered in the same manner, either in single massive doses, or in smaller quantities at frequent intervals. About three years ago, I took two ounces of it, in doses of half a teaspoonful to a teaspoonful once a day. I have also taken smaller doses more frequently, the effects being precisely similar to those produced by the soda salt. Salicylate of potash is to be preferred whenever it is desirable to introduce potash into the system rather than soda. This is often the case in the gouty diathesis, in some forms of dyspepsia, in the various manifestations of lithiasis, and in some other conditions.

Salicylate of Lithia is the next preparation I would introduce. is not so easily made; or, at any rate, my early experiments in small quantities were not very satisfactory. I therefore asked Messrs. Blake, Sandford, and Blake, to make some. At first, the slightest variations in the process gave rise to an unexpected variety in the product; but now, a salicylate of lithia of uniform appearance and quality may be obtained. I recommend it in preference to either the soda or the potash salt when it is desirable to administer a salicylate to gouty patients, or to persons suffering from the presence of uric acid. In fact, where lithia is in. dicated, it may be given in this form, provided it is desirable to give the salicylic acid, which latter may, in other cases, be given at the same time that lithia in some other form is being taken; as, e.g., during a course of lithia water.

Salicylate of Lime. - In consequence of the affinity of salicylic acid for lime the teeth may be acted on, and a mistrust has been expressed lest even the bones might suffer from a long continuance of the remedy. To meet such an objection the hypothesis may be ventured that this salt would best shield the osseous system.

Salicylate of Quinia. This salt has also been used with considerable success, particularly in those cases in which the effects of the bases already mentioned are not required, and in which a tonic, rather than a depressant, effect is desired; in fact, in those cases in which the two components of the drug are both indicated. In such cases, I have administered quinine and salicylic acid alternately; but the salicylate of quinia offers a more simple and elegant mode of prescribing, and has been well spoken of by Dr. Hewan in neuralgia and rheumatic pains.

Salicylate of Cinchonidia.-I now introduce this salt as a less costly substitute for that of quinia, and very useful as a tonic and antiperiodic in neuralgia, rheumatism, sciatica, etc. In such cases, five grains may be given every two hours, or ten grains may be given at once, and afterwards three or four doses of five grains at intervals of two or three hours. Cinchonidia $\left(\mathrm{C}_{20} \mathrm{H}_{24} \mathrm{~N}_{2} \mathrm{O}\right)$ is an isomer of cinchonia, but possesses left instead of right polarisation, and is rather more soluble. The medical committee which investigated the subject for the Government of India estimated that cinchonidia is only slightly less efficicious than quinia in fever; many observers have considered it of equal value in neuralgia. It might, therefore, be expected, that a combination of this alkaloid with salicylic acid would prove valuable in various nervous and rheumatic affections. The salicylate contains about a third of its weight of the acid, and is, of course, incompatible with iron. It is rather insoluble, and therefore the bitter taste is not quickly perceived; it may be given as a powder in wafer-paper or in a pill; or it may be suspended in a liquid, though this is not an agreeable method of taking it. I give the solid drug in the form of Wyeth's compressed tablets, which can be swallowed quickly like pills. They are sufficiently insoluble not to be tasted in this way, but they readily disintegrate in the stomach; for I have known a couple of tablets produce, in a short time, singing in the ears, which could not be distinguished from the effect of quinine by a patient who had often taken that drug. A patient who suffered acutely with neuralgia of the fifth nerve, which had been arrested twice by gelseminum, took for the third attack two tablets, repeating the dose in an hour; this sufficed. I have given it in pleurodynia and in the pains of chronic rheumatism. I have not yet tried it in acute rheumatism; but so many cases in which salicylate of soda is being employed would apparently do better if the effect of quinia in the system were substituted for that of soda, that we may hope the salicylate of cinchonidia may obtain a fair trial. Still, it is in more chronic cases of nervous and rheumatic pain, and perhaps in some of the consequences of malaria, that the chief use of this salt will probably be found. As a stimulant to appetite, and general tonic, it may also be given in moderate doses for a longer period, say two and a half to five grains twice or three times a day.

Lytham.-There is but little to note in Dr. Pountney's report for I 880 on this sea-side resort, except that there was a considerable diminution in the mortality from diseases of the respiratory organs, as compared with 1879 , and very little prevalence of infectious disease. This happy state of affairs, however, must not be expected always to last ; and it would be a wise course, on the part of the authority, to erect a hospital, to which the earliest cases of any infectious disease imported into the place could be isolated, before the infection had time to spread. The amount of sickness was below the average in every quarter, except the third, when there was prevalence of respiratory diseases and diarrhœa. The births numbered 1 23, or 24.6 per 1,000 ; the deaths 83 , or 16.6 per 1,000 , as compared with 17.8 in 1879 . Twelve of the deaths were those of visitors. 